\title{
Arm Skin
}

National Cancer Institute

\section{Source}

National Cancer Institute. Arm Skin. NCI Thesaurus. Code C52754.

The skin or integument covering the arm. 